\title{
Exploiting the Migdal effect to improve the sensitivity to light dark matter
}

\section{G. Grilli di Cortona, ${ }^{a}$ A. Messina ${ }^{b, c, *}$ and S. Piacentini ${ }^{b, c}$}

${ }^{a}$ Istituto Nazionale di Fisica Nucleare, Laboratori Nazionali di Frascati, C.P. 13, 00044 Frascati, Italy

${ }^{b}$ Dipartimento di Fisica Sapienza Università di Roma, Roma, I-00185, Italy

${ }^{c}$ Istituto Nazionale di Fisica Nucleare, Sezione di Roma, Roma, I-00185, Italy

E-mail: andrea.messina@uniroma1.it

To date, the search for dark matter weakly interacting massive particles with noble elements has set stringent constraints on masses down and below a $\mathrm{GeV} / \mathrm{c}^{2}$. The ultimate limit is represented by the experimental threshold on the energy transfer to the nuclear recoil. Currently, the experimental sensitivity has reached a threshold equivalent to a few ionization electrons. In these conditions, the contribution of a so-called Migdal electron due to the sudden acceleration of a nucleus after a collision might be sizeable. We present a recent work where, using a Bayesian approach, we studied how these effects can be exploited in experiments based on liquid argon detectors. We develop a simulated experiment to show how the Migdal electron allows to push the experimental sensitivity down to masses of $0.1 \mathrm{GeV} / \mathrm{c}^{2}$, extending the search region for dark matter particles of previous results. Finally, given the relevance of the Migdal electrons to the search for low mass dark matter, we discuss some new ideas on how to possibly measure such an effect with detectors based on a time projection chamber exposed to a high neutron flux.

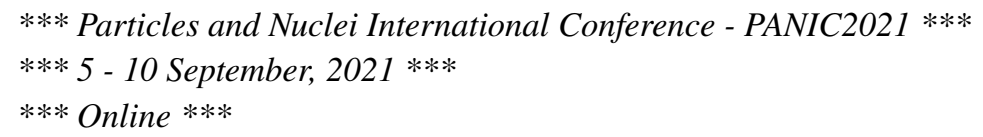

${ }^{*}$ Speaker 


\section{Introduction}

The presence of dark matter is supported by compelling gravitational evidence at all length scales, from galaxies to the whole universe. However, the experimental effort aimed at revealing the mass of its elementary constituents and its interaction with ordinary matter has not provided a definitive answer.

Direct detection experiments look for the recoil energy deposited by an elastic scattering of $\mathrm{DM}$ on atomic nuclei. In this process, one is typically interested in the kinetic energy acquired by the nuclear recoil (NR), disregarding the behavior of the electron cloud. The interaction rate can be parameterized in terms of DM mass and DM-nucleon cross section, and these two parameters are in turn used to report the experimental sensitivity. For large DM masses (with respect to the atomic mass of the target) the sensitivity scales with the experiment exposure, while for smaller masses the ultimate limit is represented by the energy threshold, typically of the order of $1 \mathrm{keV}$. A great effort is currently being invested in refining the experimental techniques to reach sub-keV energy thresholds. In addition, subleading interaction channels, disregarded so far, can be exploited to increase the experimental sensitivity to light DM. This work elaborates on this theme, studying the contribution of the so-called Migdal Effect (ME) [1,2] to the search of light DM with liquid argon (LAr) experiments. We present results of a simulation that show how the ME allows to push the experimental sensitivity down to masses of $0.1 \mathrm{GeV} / \mathrm{c}^{2}$ [3] extending the search region for DM of previous results. Finally, given the relevance of the ME to the search for low mass DM, we discuss some new ideas on how to possibly measure such an effect with detectors based on a Time Projection Chamber (TPC) exposed to a high neutron flux.

\section{The Migdal effect}

At small momentum transfers it is relevant to accurately describe the behavior of the electrons during the DM scattering with the nucleus. The collision causes a perturbation of the atomic system, that leads to a small, but non-zero, transition probability from the initial ground state to ionized states. Therefore, thanks to the Migdal effect, the final state might contain one or more free electrons in addition to the initial nuclear recoil. This is the ME. The kinematic of the process implies the following relations between the DM velocity and the energy $E_{R}$ and $E_{d}$ transferred respectively to the the NR and the electron:

$$
v_{\min }=\sqrt{\frac{m_{N} E_{R}}{2 \mu_{N}^{2}}}+\frac{E_{d}}{\sqrt{2 m_{N} E_{R}}}, \quad E_{R}^{\max }=\frac{2 \mu_{N}^{2} v_{\max }^{2}}{m_{N}}, \quad E_{d}^{\max }=\frac{\mu_{N} v_{\max }^{2}}{2},
$$

where $E_{R}$ is the nuclear recoil energy, $E_{d}$ is the detected energy of the electron, $\mu_{N}$ is the DM nucleus reduced mass and $m_{N}$ is the nucleus mass.

Figure 1 gives the maximum energy $E_{R}$ for the nuclear recoil (solid curves) and $E_{d}$ for the $\mathrm{ME}$ (dashed curves) as a function of the DM mass for a number of atomic targets. Considering a detection threshold of $1 \mathrm{keV}$, the nuclear recoil on argon goes below threshold around $2 \mathrm{GeV}$, while including the ME allows access to smaller masses. This is due to the fact that the maximum NR energy gets a suppression factor of $\mu_{N} / m_{N}$ with respect to the electron energy. In conclusion, at low DM masses it is easier to detect an electron from the ME rather than a NR. 

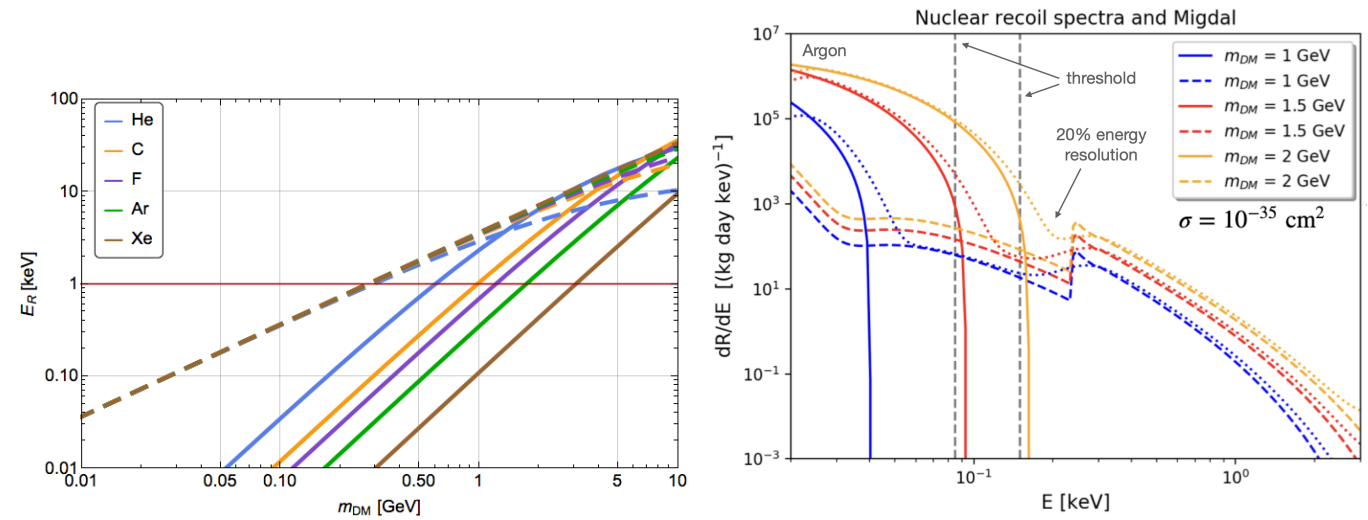

Figure 1: (Left) Kinematic limits of the nuclear recoil (solid) and ME (dashed) for different atoms: He (blue), C (orange), F(purple), Ar (green) and Xe (brown). (Right) Spectra of the NR and ME for an isolated argon atom for different DM masses.

The differential rate of ionization due to the ME for a nuclear recoil energy $E_{R}$ accompanied by a ionization electron with energy $E_{e}$ is given by [2]

$$
\frac{d^{2} R}{d E_{R} d E_{e}}=\frac{d R}{d E_{R}}\left|Z\left(E_{R}, E_{e}\right)\right|^{2}, \quad\left|Z\left(E_{R}, E_{e}\right)\right|^{2} \simeq 1+\frac{1}{2 \pi} \sum_{n, \ell} \int d E_{e} \frac{d p_{q_{e}}^{c}\left(n \ell \rightarrow E_{e}\right)}{d E_{e}}
$$

where $d R / d E_{R}$ is the usual DM nuclear recoil rate, while $\left|Z\left(E_{R}, E_{e}\right)\right|^{2}$ is the term that takes into account the ionization rate (the de-excitation is negligible). The probability $p_{q_{e}}^{c}$ to emit an electron ${ }^{1}$ was computed for several isolated atoms in Ref. [2]. The differential rate to emit a ME electron (dashed curves) and that of the usual NR process (solid curves) for an argon isolated atom is shown in Fig. 1.

\section{Impact of the Migdal effect on the DM sensitivity of LAr experiments}

To quantify the possible impact of considering the ME in existing and planned LAr TPC experiments, we used a toy experiment called TEA-LAB based on a LAr TPC. To perform this study we used the signal templates for the NR and the ME discussed in the previous section, and we relied on published experimental work [4] for a parameterization of detector response and the background spectra. Finally, we performed a Bayesian analysis to extract the projected sensitivity.

We employed to following likelihood function $\mathcal{L}=\mathcal{L}_{C} \times \mathcal{L}_{\mathcal{B}} \times \mathcal{L}_{\mathcal{S}}$, factorized in three terms:

$$
\mathcal{L}_{C}=\prod_{i=1}^{N_{\text {bin }}} \frac{\lambda_{i}^{x_{i}}}{x_{i} !} e^{-\lambda_{i}}, \quad \mathcal{L}_{\mathcal{B}}=\prod_{\{\text {bkg }\}} \prod_{i=1}^{N_{\text {bin }}} \mathcal{N}(\mu, \sigma), \quad \mathcal{L}_{\mathcal{S}}=f\left(S_{i}, \epsilon\right) .
$$

$\mathcal{L}_{c}$ describes the probability that in each observation bin $i$ the number of counts is $x_{i}$. It is assumed to be Poissonian given the expected number of counts $\lambda_{i}=E\left[r_{S} S_{i}+r_{B} B_{i}\right]$, with the counts taken independent in different bins. $E$ is the experimental exposure, while the quantities $S_{i}$ and $B_{i}$ are associated respectively to the DM signal and the total background. $\mathcal{L}_{B}$ and $\mathcal{L}_{S}$ describe how the background and the signal templates are affected by systematic uncertainties.

\footnotetext{
${ }^{1} n$ and $\ell$ are initial quantum numbers, $E_{e}$ is the final electron energy, and $q_{e}$ is the electron momentum in the nucleus rest frame.
} 


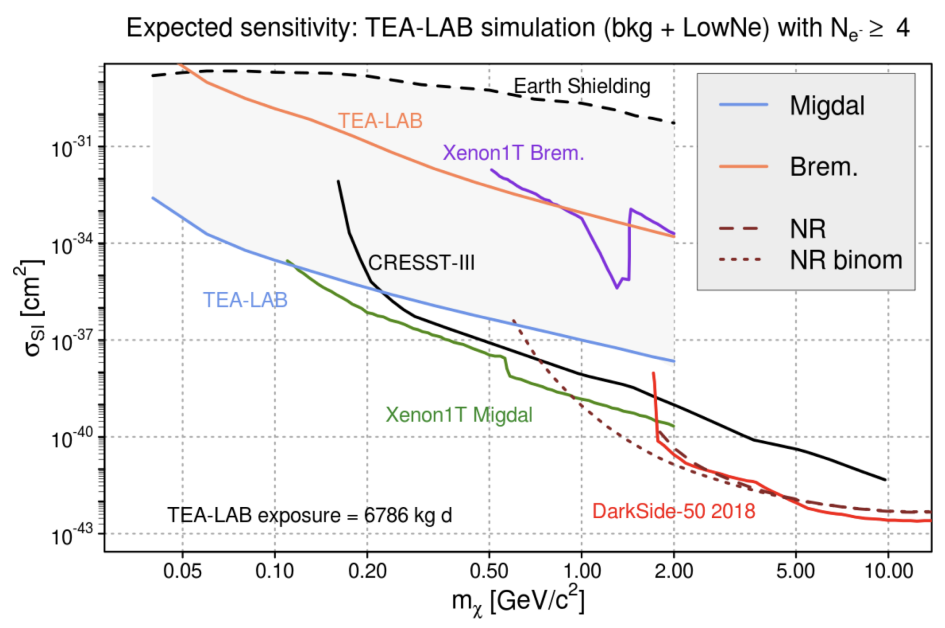

Figure 2: Expected sensitivity on the $\sigma_{S I}$ exploiting the ME for th TEA-LAB simulated experiment using an exposure of $6786 \mathrm{~kg}$ day (blue). The red curve is the current DarkSide-50 bound [4], the green is the bound from the Xenon1T Migdal search [5] and the black one is the bound by the CRESST-III experiment [6].

Fig. 2 shows the expected sensitivity of our TEA-LAB simulated experiment. The red curve is the result by the Darkside collaboration while the dashed brown curve is the validation of our analysis on that result, the green curve is the Migdal result by the Xenon1T collaboration, and finally the blue curve is the sensitivity of the TEA-LAB simulation with an exposure of $6786 \mathrm{~kg}$ day. It is possible to notice that this result is comparable with the Xenon1T one and it even surpasses its sensitivity for dark matter masses below $\sim 0.1 \mathrm{GeV}$. These are promising results, but the ME has not yet be observed in nuclear scattering.

\section{Prospects to observe the Migdal effect in nuclear recoils}

To observe the ME in NR there are two interesting signatures that can be exploited. The first one relies on fast neutrons to induce an energetic NR and with probability $O\left(10^{-4}\right)$ a ionization of an electron from the innermost shell. The atom will then fill the hole by emitting a monochromatic $\mathrm{x}$-ray that for $\mathrm{Ar}$ is at $3 \mathrm{keV}$. This will give rise to a interesting event tag represented by an energetic $\mathrm{NR}$ and an electromagnetic energy deposit of $3 \mathrm{keV}$ few centimeters away from the NR. The second option consists in detecting the ME electron, exploiting the contribution from all atomic shells, together with the recoiling nucleus. In this case, one needs good tracking capabilities to reconstruct a nuclear and electron recoil originating from the same vertex.

Such events can actually be seen by current direct detection experiments. In particular the CYGNO TPC [7] based on a gas mixture of He/Ar CF4 at atmospheric pressure with optical readout is a very good candidate. We have the possibility to use a high intensity neutron source at 2.5 or $14 \mathrm{MeV}$. We are currently working on defining the optimal detector configuration for a dedicated Migdal run.

We expect for the X-ray signature the following number of events,

$$
N_{\text {events }}=N_{T} \Phi \sigma_{\mathrm{Ar}} f_{\mathrm{Ar}} \mathrm{BR}_{\mathrm{ME}} \simeq 400 \mathrm{day}^{-1},
$$


where $N_{T}$ is the number of target nuclei, $\Phi$ is the flux for a $2.5 \mathrm{MeV}$ neutron source at a distance of around $3 \mathrm{~m}, \sigma_{\mathrm{Ar}}$ is the n-Ar scattering cross section for a $2.5 \mathrm{MeV}$ neutron, $f_{\mathrm{Ar}}$ is the fluorescence yield, namely the probability of de-excitation of an excited atom (an atom with a core-hole) by emitting a fluorescence photon. The fluorescence yield is $\sim 0.14$ in Ar. Finally, $\mathrm{BR}_{M E}$ is the Migdal branching ratio (considering only the most internal shell). The expected exponential distribution for absorption length of the X-ray should help to reject flat neutron multiple scattering or the distribution for accidental backgrounds. Concerning the Migdal electron signature, one needs to be able to reconstruct nuclear recoils and electron recoils starting from the same vertex. This should be possible for large nuclear recoil energies and for electron recoil energies down to $\sim 5-10$ $\mathrm{keV}$. Integrating the double differential event rate from a NR energy of $100 \mathrm{keV}$ to $E_{R}^{\max }$ and from $E_{e}$ of $5 \mathrm{keV}$ to $E_{e}^{\max }$ we obtain $\sim 600$ events per day in a mixture 60:40 of $\mathrm{Ar}_{\mathrm{r}} \mathrm{CF}_{4}$ and a neutron source of $2.5 \mathrm{MeV}$. These event yields confirm the possibility to study the ME in nuclear recoil events with the present TPCs, however a detailed simulation of the background levels and the reconstruction efficiency is needed to confirm the feasibility of these measurements.

\section{Conclusions}

We showed that the Migdal effect has a great potential to improve the current sensitivity to light DM exploiting LAr detector. To rely on these results it is compelling to observe the Migdal effect in NR. There are promising signatures to be exploited using fast neutrons and the CYGNO TPC is an interesting experimental opportunity on which we are currently working to identify the best configuration.

\section{References}

[1] A. Migdal, Ionization of atoms accompanying $\alpha$ - and $\beta$-decay, J. Phys. USSR 4 (1941) 449.

[2] M. Ibe, W. Nakano, Y. Shoji, and K. Suzuki, Migdal Effect in Dark Matter Direct Detection Experiments, JHEP 03 (2018) 194, [arXiv: 1707 . 07258].

[3] G. Grilli di Cortona, A. Messina, and S. Piacentini, Migdal effect and photon Bremsstrahlung: improving the sensitivity to light dark matter of liquid argon experiments, JHEP 11 (2020) 034, [arXiv: 2006.02453].

[4] DarkSide Collaboration, P. Agnes et al., Low-Mass Dark Matter Search with the DarkSide-50 Experiment, Phys. Rev. Lett. 121 (2018), no. 8 081307, [arXiv: 1802. 06994].

[5] XENON Collaboration, E. Aprile et al., Search for Light Dark Matter Interactions Enhanced by the Migdal Effect or Bremsstrahlung in XENONIT, Phys. Rev. Lett. 123 (2019), no. 24 241803, [arXiv: 1907.12771].

[6] CRESST Collaboration, A. Abdelhameed et al., First results from the CRESST-III low-mass dark matter program, Phys. Rev. D 100 (2019), no. 10 102002, [arXiv: 1904. 00498].

[7] E. Baracchini et al., Identification of low energy nuclear recoils in a gas time projection chamber with optical readout, Measur. Sci. Tech. 32 (2021), no. 2 025902, [arXiv:2007.12508]. 